\title{
Immediate effects of two different ankle taping techniques on ankle inversion during quick lateral cutting movements; a pilot crossover randomised controlled trial.
}

\author{
Koya Mine ${ }^{1,2 *}$, Keio Ishiguro ${ }^{1}$, Takashi Nakayama ${ }^{1,2}$ \\ ${ }^{1}$ Department of Physical Therapy, School of Health Sciences, Tokyo University of Technology, 5-23-22 Nishi-kamata, \\ Ota ward, Tokyo, 144-8535, Tokyo, Japan \\ ${ }^{2}$ International Centre for Allied Health Evidence, University of South Australia, Adelaide, South Australia, Australia
}

\begin{abstract}
The objective of this pilot study was to examine and compare immediate effects of traditional ankle taping (TAT) and Mulligan ankle taping (MAT) on ankle inversion during quick lateral cutting movements. This study was a pilot crossover randomised controlled trial in a laboratory setting. 12 healthy collegiate male students (age $21.8 \pm 0.5$ years, body mass index $22.6 \pm 1.8$ ) received the two different taping techniques in a random order. A six-camera Vicon motion-analysis system and one force plate were used to assess peak ankle inversion angle and moment, and performance of the repetitive quick lateral cutting movement task before and after interventions. Neither of the two groups showed significant change after taping applications compared to the baselines in any outcome measure. Mean changes in all outcome measure were not significantly different between the two interventions groups. In conclusion, the application of TAT or MAT might not immediately change ankle inversion kinematics or kinetics, or performance of quick lateral cutting movements of healthy young male adults.
\end{abstract}

Keywords: Ankle taping, Mulligan taping, Lateral cutting movements, Ankle inversion.

Accepted on December 14, 2017

\section{Introduction}

Lateral ankle sprain (LAS) is one of the most common acute injuries in many weight-bearing sports, such as basketball, football and volleyball [1]. This injury has a high recurrence rate, which can make injured athletes vulnerable to chronic problems [2]. Following LAS, $10-30 \%$ of athletes are reported to have persistent symptoms or reinjuries [3]. It has been reported that individuals with chronic ankle instability may suffer from pain, diminished neuromuscular control, muscle weakness, impaired joint position sense and compromised performance during functional movements [4]. These physical impairments can prevent athletes with chronic ankle instability from full recovery and return to sports. Due to its high prevalence and recurrence rate, its economic seems to be substantially large [1]. Therefore, prevention of LAS is an important issue in the field of clinical sports science.

The pathophysiology of LAS involves sprain or complete rupture of anterior talofibular ligament and/or calcaneofibular ligament (2002). These ligaments can be mechanically stretched and damaged through excessive inversion of the ankle joint [5]. This excessive ankle inversion is often induced during quick lateral cutting movements in sports participation [6,7]. Recent evidence suggests that there may be a positional shift of fibula among patients with subacute LAS or chronic ankle instability. Some studies found that fibula tended to be positioned anteriorly after injuries [8,9]. Although the mechanism of the positional alteration as pathophysiology of
LAS is still elusive, these findings became a rationale of some treatment strategies, which aims to correct the fibular positional change, and improve pain and ankle range of motions among patients with LAS [10,11]. Since the positional change is thought to be a primary pathology to be corrected, it is described as 'positional fault' by its advocates $[8,9]$.

Prophylactic taping techniques are commonly performed for athletes before sports events in order to prevent LAS. The most conventional technique involves stirrups with horseshoe and heel locks to restrict both talocrural and subtalar motions, particularly inversion [12]. This traditional ankle taping technique (TAT) is based on a straightforward mechanistic idea regarding the pathophysiology of LAS, and has been reported to be effective to prevent LAS [13]. Despite its clinical effectiveness, few data exist to help us understand how TAT can act to control ankle inversion kinematics and kinetics during dynamic activities, such as lateral cutting movements and running [14]. Mulligan ankle taping (MAT), another kind of taping technique is applied to shift the fibula to the posterosuperior direction. This taping technique is based on the positional fault theory as mentioned the above, and has been reported to be potentially effective to prevent LAS in basketball $[11,15]$. To date, there has been no study comparing the immediate effects of these two taping methods on ankle kinematics and kinetics during quick lateral cutting movements.

The purpose of this study was to examine and compare immediate effects of the two different types of taping 
Citation: Koya M, Ishiguro K, Nakayama T. Immediate effects of two different ankle taping techniques on ankle inversion during quick lateral cutting movements; a pilot crossover randomised controlled trial. J Phys Ther Sports Med 2018;2(1):5-9.

techniques on ankle inversion kinematics and kinetics during quick lateral cutting movements. We also aimed to establish a protocol for testing through this pilot study. Secondary outcome measure was a performance of the cutting movement task. It was hypothesised that TAT would be more effective to restrict ankle inversion movements and moment, based on its apparent mechanical advantage.

\section{Methods}

\section{Participants}

An ethical approval was achieved from an ethical committee at Tokyo University of Technology before the commencement of the experiments (registration number: E16HS-032). Subjects were recruited from undergraduate physiotherapy students at Tokyo University of Technology. Inclusion criteria were: (1) healthy men aged over 20 years, (2) no history of ankle injury in the last one year, (3) no history of ankle surgery, (4) no significant ankle laxity and (5) no pain during quick lateral cutting with maximal effort. Significant ankle laxity, implying grade-1ll LAS in the past, was identified through two orthopaedic tests, including talar tilt test and anterior drawer test [16]. Excessive movements in ankle inversion and anterior glide of talus were regarded as positive in the two manual examinations respectively. The screening procedures were administered by the same physiotherapist (KM), who had completed Masters degree in musculoskeletal and sports physiotherapy. Before participation, all subjects were informed of the aims and risks of the study and gave written informed consent.

\section{Measures}

A six-camera Vicon motion-analysis system and Vicon Nexus software version 1.7.1 (Vicon, UK) were utilised to evaluate and process the kinematic and kinetic data of the participants. A camera frequency was set at $100 \mathrm{~Hz}$. In testing sessions, a total of 35 reflective markers $(1.4-\mathrm{cm}$ diameter) were attached at bony landmarks of the whole body, based on the Plug-InGait model (Vicon, UK) (see Figure 1). Participants were instructed to wear only non-reflective firm-fitting elastic short pants. All the tasks were performed barefoot. The raw data of marker positions were digitally filtered with a fourth order low-pass Butterworth filter with $6 \mathrm{~Hz}$ cut-off frequency. The motion-analysis system was operated by the same physiotherapist (KM).

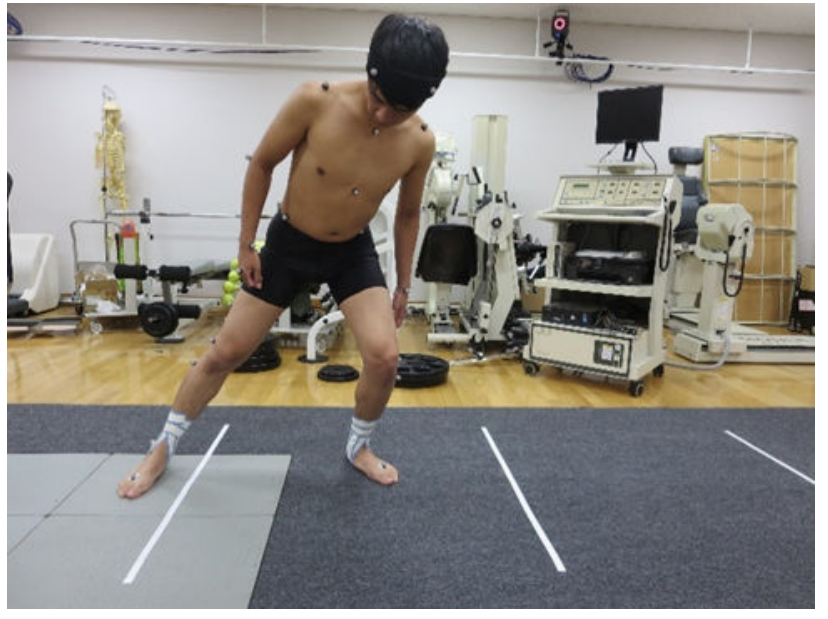

Figure 1. Assessment of the quick lateral cutting movement task using VICON.

Before the commencement of the trial, participants were instructed to shave the lower one third of their shin to optimise the contact of the tape and skin. In first experimental sessions, anthropometric measurements were conducted. After all markers were positioned on the given bony landmarks, subjects performed quick lateral cutting once with maximal efforts to familiarise themselves with the task. It also served the purpose as a warm-up. In the task, subjects were instructed to repeat side steps as quickly as possible over three horizontal lines for 10 seconds (see Figure 2). The lines were set with white adhesive tapes (Nitto, Japan) with one-metre distance from each other (see Figure 2). Participants started the lateral cutting task to the right side immediately after the assessor's verbal cue "ready, go", and then stopped after another cue "stop" 10 seconds later. Both verbal cues were spoken in Japanese language by the same assessor (KM). The line on the right side was set on a single force plate (BP400600-2000; AMTI, USA) to measure the ground reaction force during weight bearing of dominant feet (see Figure 1 and 2). Kinematic and kinetic data was extracted from initial foot contact to toe-off of every step on the dominant side during the 10 -second measurement period. Mean values for peak ankle inversion angle and peak ankle inversion moment were calculated subsequently. Furthermore, the number of lines crossed over by subjects in 10 seconds were manually counted to assess the performance of the task.

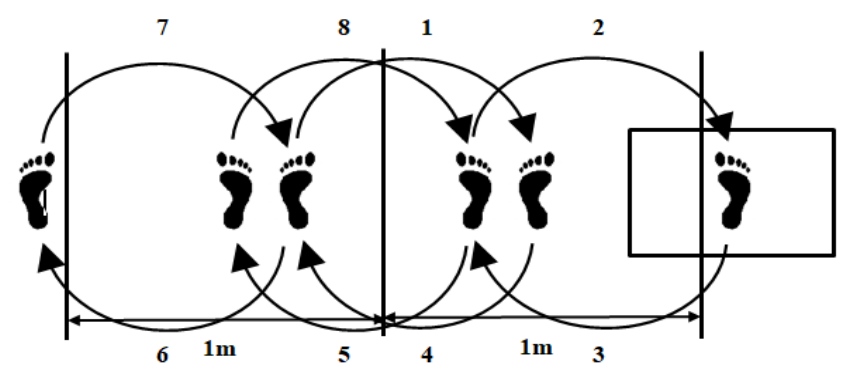

Figure 2. Stepping manner in the task.

Following the warm-ups, subjects rested on a chair with back rest for six minutes. After subjects performed the task as the 
baseline assessment, they randomly received either TAT or MAT applications. Non-elastic white and blue tape $(3.8 \mathrm{~cm}$ width) was used for all interventions (Nitto, Japan). TAT consisted of one anchor, three stirups with horseshoe, figure six and reverse figure six, heel lock and reverse heel lock, and one anchor on top of them (Figure 3). MAT was administered as suggested by advocates of Mulligan techniques to facilitate posterosuperior glide of fibula (Figure 3) [11]. In the application of MAT, tapes were also applied on heels to ensure the comparability between two different taping techniques and eliminate its effects on ankle kinematics and kinetics (Figure 3). Taping techniques were applied for both legs in a longsitting position. It took six minutes for the physiotherapist (KM) to complete TAT for both sides, whereas four minutes in MAT. After MAT applications, subjects were told to rest for another two minutes in the long-sitting position. After the application of taping, quick lateral cutting movements were assessed in the same manner as the baseline measurements.
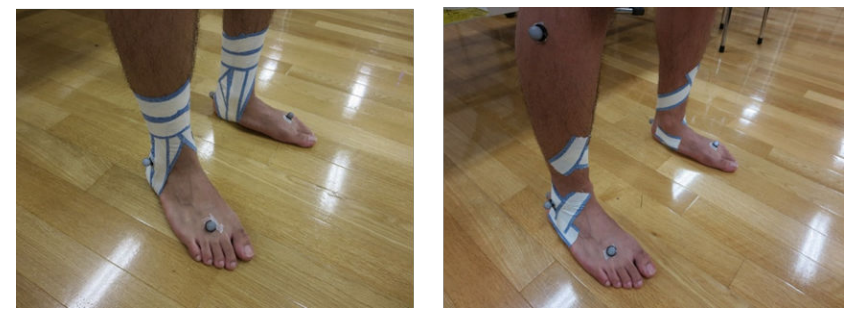

Figure 3. Two types of taping interventions; TAT (left) and MAT (right).

Two testing sessions were separated by 48 hours at least, in order to minimise the effects of fatigue and delayed onset muscle soreness in lower-limb muscles. Subjects were instructed to refrain from alcohol intake for 24 hours before testing sessions and not to participate in vigorous exercises for 48 hours before sessions. All testing sessions were conducted in the same laboratory room at the same temperature of 24 degrees Celsius. A care was taken to ensure that all participants received consistent taping applications, verbal instructions and visual cues to minimise potential influences on arousal levels of the subjects.

\section{Design and procedures}

This study was a crossover randomised controlled trial, in which all eligible subjects completed two days of experiments in a random order. This research design was chosen in order to ensure as many participants as possible. A trial protocol was registered in University Hospital Medical Information Clinical Trials Registry (registration number: UMIN000025985) in advance. Each experimental session consisted of a warm-up, applications of TAT or MAT, and pre- and post-intervention assessments of quick lateral cutting movements. Due to the nature of the taping interventions, it was not possible to blind participants or therapists. The same physiotherapist (KM) performed both taping applications and assessments of the task. After the inclusion of participants, they performed two experiment sessions in a random order, which guaranteed the allocation concealment [17].

\section{Statistical analysis}

The results are presented as mean \pm standard deviation (SD) values. The baseline data was utilised to calculate intraclass correlation (ICC) and kappa statistics, and determine the reliability of the outcome measures. ICC and kappa scores were evaluated accordingly: $<0.20$ as slight, $0.21-0.40$ as fair, $0.41-0.60$ as moderate, $0.61-0.80$ as substantial and $0.80<$ as almost perfect [18]. A paired t-test was used to assess differences between pre-test and post-test within each condition, and differences between the two conditions. Differences were considered to be statistically significant at $\mathrm{p}<0.05$. All statistical tests were conducted with SPSS (IBM, USA). Hedges' $g$ and 95\% confidence intervals (CI) were calculated to determine within-group effect sizes [19]. Effect size was categorised as large $(\geq 0.80)$, moderate $\geq 0.50)$ or small ( $\geq 0.20$ ) [20]. A post-hoc sample-size calculation was performed using G-Power 3.1.9.2 (University of Dusseldorf, Germany), where an alpha level was set at 0.05 .

\section{Results}

The screening process is illustrated in Figure 4. As a result of the screening, 12 healthy male collegiate students (age $21.8 \pm$ 0.5 years, body weight $65.5 \pm 7.8 \mathrm{~kg}$, height $170.2 \pm 5.1 \mathrm{~cm}$, body mass index $22.6 \pm 1.8$ ) met the inclusion criteria and were recruited for this study. All participants were right leg dominant. Current exercise habits of participants were varied; twice per week $(n=4)$, once per week $(n=5)$, once per fortnight $(n=2)$ and none at the moment $(n=3)$. All participants completed both experimental sessions without any injuries and there was no drop out. None of the subjects accidentally fell or collapsed during the task.

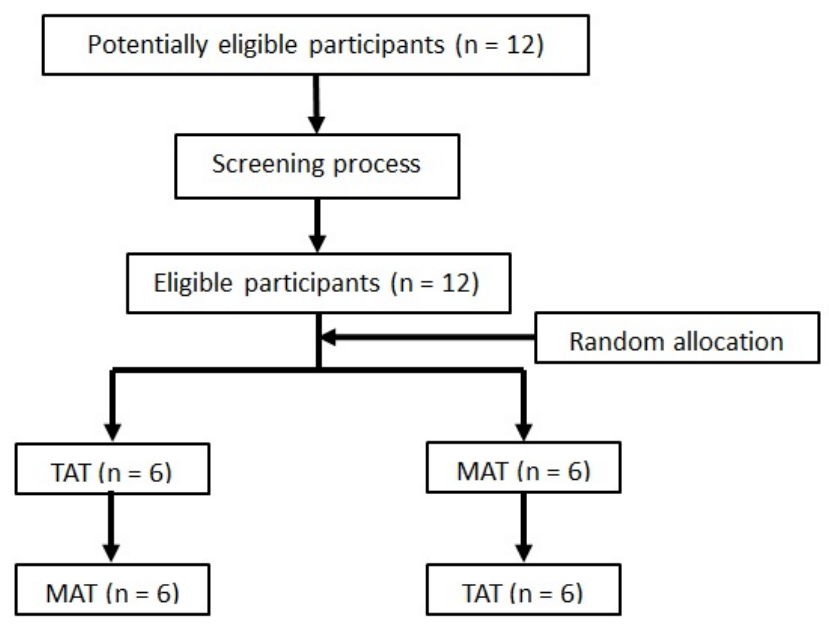

Figure 4. Flowchart of the study.

At baseline, there was no significant difference in any outcome measures between TAT and MAT groups (all $\mathrm{p}>0.33$ ), which guaranteed the baseline comparability of the two groups (refer to Table 1). Further analysis of the baseline data proved substantial to almost perfect reliability of the outcome measures, with ICC being 0.75 and 0.90 for peak ankle inversion angle and moment respectively.[18] The reliability to 
Citation: Koya M, Ishiguro K, Nakayama T. Immediate effects of two different ankle taping techniques on ankle inversion during quick lateral cutting movements; a pilot crossover randomised controlled trial. J Phys Ther Sports Med 2018;2(1):5-9.

assess the overall performance was almost perfect with kappa score of 0.95 .

As a result of within-group comparisons, there was no immediate significant change in any outcome measures in the two groups (all p>0.37, refer to Table 1). Between-group comparisons revealed no significant difference in the changes in any outcome measures (all $\mathrm{p}>0.38$ ).
Within-group effect sizes were consistently small $($ all $<0.20)$ and $95 \%$ CI included the value of zero, which implied no clinical significance of the effects (refer to Table 1). As a result of a post-hoc calculation, it was estimated that the statistical power ranged from 0.05 (performance in TAT group) to 0.28 (performance in MAT group).

Table 1. A comparison of peak ankle inversion angle and moment. Values for performance were described as median.

\begin{tabular}{|c|c|c|c|c|c|c|c|c|}
\hline \multirow{2}{*}{ Outcome measure } & \multicolumn{2}{|l|}{ TAT $(n=12)$} & \multirow[t]{2}{*}{$\mathrm{p}$} & \multirow[t]{2}{*}{ Effect size } & \multicolumn{2}{|c|}{ MAT $(n=12)$} & \multirow[t]{2}{*}{$\mathrm{p}$} & \multirow[t]{2}{*}{ Effect size } \\
\hline & pre & post & & & pre & post & & \\
\hline Peak Inversion Angle $\left[{ }^{\circ}\right]$ & $5.4 \pm 3.3$ & $5.0 \pm 3.4$ & 0.45 & 0.09 & $4.8 \pm 3.3$ & $4.4 \pm 3.9$ & 0.47 & 0.11 \\
\hline $\begin{array}{l}\text { Peak Inversion Moment } \\
{[\mathrm{Nm}]}\end{array}$ & $0.75 \pm 0.21$ & $0.72 \pm 0.14$ & 0.38 & 0.07 & $0.71 \pm 0.19$ & $0.73 \pm 0.19$ & 0.62 & -0.08 \\
\hline Performance [times] & 22.5 & 22 & 0.67 & 0.04 & 22.5 & 21.5 & 0.53 & -0.29 \\
\hline
\end{tabular}

\section{Discussion}

To date, to the best of the authors' knowledge, this study is the only investigation examining the acute effects of TAT and MAT on ankle inversion kinematics, kinetics, and performance in quick lateral cutting movements. Although the current evidence suggests that TAT can restrict passive ankle inversion range of motion when it is measured in a static manner, it is unclear whether it can control ankle inversion during dynamic movements [14]. There is also no clear evidence whether the application of any taping techniques can alter ankle inversion kinetics or functional performance or not [14]. Although we hypothesised that TAT might be more efficient to control ankle inversion motion and moment due to its potential mechanical advantage, the results of this research suggest that neither of the taping interventions may produces no immediate positive effect to restrict ankle inversion stress or enhance performance in quick lateral cutting manoeuvres.

The efficacy of TAT to prevent LAS among sporting population has been supported by recent systematic review [13, 21]. Considering its evidence-based effectiveness in clinical sports settings, one potential reason why there was no significant change in ankle inversion kinematics in this study could be that TAT might mechanically control abrupt endrange inversion only when accidental collapse or falls occur. Otherwise, there might be another mechanism, where applications of TAT can stimulate sensorimotor functions and contribute to more muscular stability to restrict abrupt ankle inversion. Unlike TAT, there is very limited evidence to support the benefits of MAT to prevent LAS [15]. Considering the evidence for the preventative effects of TAT for LAS, the lack of positive findings in this study does not discourage the use of TAT in sporting settings. The fact that there was no change in ankle inversion may imply that TAT might be effective to prevent LAS without compromising athletes' ankle movements and functional movements. In fact, neither of the taping techniques affected the lateral cutting task positively or negatively.
Several methodological limitations in this research have to be critically appraised. Firstly, we examined the effects of the two taping techniques on healthy male subjects with various exercise habits. Thus, the findings in this research have to be cautiously applied to other populations, especially patients with LAS or chronic ankle instability. It has to be also noted that the data might differ when the same task is performed with shoes on. Secondly, we are still uncertain about the effects of these taping techniques in other movement tasks, such as hopping or landing, which can also contribute to the onset of LAS in sports settings. Thirdly, the inability to blind the therapist and participants might have confounded the results. Lastly, posthoc sample size calculation revealed potential lack of necessary samples to achieve statistical significance. This was predictable due to the nature of the trial as a pilot study. Additional investigations with larger sample sizes and better statistical precisions are necessary. Given that effect sizes were consistently small, however, it is questionable whether collecting more samples would affect clinical significance or not. Lastly, there was no accidental falls or collapse in the experiments in this study. Thus, it is still not clear whether these taping techniques are able to mechanically restrict ankle inversion kinematics during accidental falls or collapse.

\section{Conclusion}

The application of TAT or MAT might not immediately change ankle inversion kinematics or kinetics, or performance of quick lateral cutting movements. Given the strength of evidence for TAT and MAT, TAT can be used to prevent LAS without compromising lateral cutting movement performance in sports settings. Further studies with larger sample sizes are required to investigate the effects of TAT and MAT on ankle kinematics and kinetics of patients with LAS or chronic ankle instability.

\section{Conflict of interest}

None declared. 


\section{References}

1. Waterman BR, Owens BD, Davey S, et al. The epidemiology of ankle sprains in the United States. JBJS. 2010;92(13):2279-2284.

2. Pourkazemi F, Hiller CE, Raymond J, et al. Predictors of chronic ankle instability after an index lateral ankle sprain: a systematic review. Journal of Science and Medicine in Sport. 2014;17(6):568-573.

3. Peters JW, Trevino SG, Renstrom PA. Chronic lateral ankle instability. Foot \& ankle. 1991;12(3):182-191.

4. Hale SA, Hertel J, Olmsted-Kramer LC. The effect of a 4week comprehensive rehabilitation program on postural control and lower extremity function in individuals with chronic ankle instability. Journal of Orthopaedic \& Sports Physical Therapy. 2007;37(6):303-311.

5. Hertel J. Functional anatomy, pathomechanics, and pathophysiology of lateral ankle instability. Journal of athletic training. 2002;37(4):364.

6. Gudibanda A, Wang Y. Effect of the ankle stabilizing orthosis on foot and ankle kinematics during cutting maneuvers. Research in Sports Medicine. 2005;13(2): 111-126.

7. Mckay GD, Goldie P, Payne WR, et al. Ankle injuries in basketball: injury rate and risk factors. British journal of sports medicine. 2001;35(2):103-108.

8. Hubbard TJ, Hertel J. Anterior positional fault of the fibula after sub-acute lateral ankle sprains. Manual therapy. 2008;13(1):63-67.

9. Kavanagh J. Is there a positional fault at the inferior tibiofibular joint in patients withacute or chronic ankle sprains compared to normals? Manual Therapy. 1999;4(1): 19-24.

10. Collins N, Teys P, Vicenzino B. The initial effects of a Mulligan's mobilization with movement technique on dorsiflexion and pain in subacute ankle sprains. Manual therapy. 2004;9(2):77-82.

11. Hopper D, Samsson K, Hulenik T, et al.. The influence of Mulligan ankle taping during balance performance in subjects with unilateral chronic ankle instability. Physical Therapy in Sport. 2009;10(4):125-130.

12. Callaghan MJ. Role of ankle taping and bracing in the athlete. British Journal of Sports Medicine. 1997;31(2): 102-108.
13. Dizon JMR, Reyes JJB. A systematic review on the effectiveness of external ankle supports in the prevention of inversion ankle sprains among elite and recreational players. Journal of Science and medicine in sport. 2010;13(3):309-317.

14. Cordova ML, Ingersoll CD, Palmieri RM. Efficacy of prophylactic ankle support: an experimental perspective. Journal of athletic training. 2002;37(4):446.

15. Moiler K, Hall T, Robinson K. The role of fibular tape in the prevention of ankle injury in basketball: A pilot study. Journal of Orthopaedic \& Sports Physical Therapy. 2006;36(9):661-668.

16. Docherty CL, Rybak Webb K. Reliability of the anterior drawer and talar tilt tests using the LigMaster joint arthrometer. Journal of sport rehabilitation. 2009;18(3): 389-397.

17. Schulz KF, Grimes DA. Allocation concealment in randomised trials: defending against deciphering. The Lancet. 2002;359(9306):614-618.

18. Landis JR, Koch GG. An application of hierarchical kappatype statistics in the assessment of majority agreement among multiple observers. Biometrics. 1977:363-374.

19. Hedges LV. Estimation of effect size from a series of independent experiments. Psychological bulletin. 1982;92(2):490.

20. Cohen J. Statistical power analysis for the behavioral sciences Lawrence Earlbaum Associates. Hillsdale, NJ. 1988:20-26.

21. Evans LJ, Clough A. Prevention of ankle sprain: A systematic review. International Musculoskeletal Medicine. 2012;34(4):146-158.

\section{*Correspondence to:}

Koya Mine

Department of Physical Therapy

School of Health Sciences, Tokyo University of Technology

5-23-22 Nishi-kamata, Ota ward

Tokyo, Japan

E-mail: mineky@stf.teu.ac.jp 